\title{
Correlation between ischemia-modified albumin and lipid levels in patients with acute cerebrovascular disease
}

\author{
KUN HAN, NING JIA, LI YANG and LIAN-QIU MIN \\ Department of Neurology, The First Affiliated Hospital of Liaoning Medical College, Jinzhou, Liaoning 121001, P.R. China
}

Received February 12, 2012; Accepted June 15, 2012

DOI: $10.3892 / \mathrm{mmr} .2012 .961$

\begin{abstract}
Acute cerebrovascular disease (ACVD) is associated with high morbidity and mortality rates, however, molecular markers to aid in its early detection are lacking. In this study, we examined the correlation between serum ischemia-modified albumin (IMA), a marker used to identify ischemic events in the heart, and blood lipids in patients with ACVD. Serum IMA levels were determined by enzyme-linked immunosorbent assay, and total cholesterol (TC), low-density lipoprotein (LDL), high-density lipoprotein cholesterol (HDL) and triglyceride (TG) levels were determined biochemically in 62 patients with cerebral infarction (CI), 40 patients with intracerebral hemorrhage $(\mathrm{ICH}), 18$ patients with subarachnoid hemorrhage (SAH) and 100 healthy individuals (controls). Serum IMA levels were significantly higher in each of the ACVD groups compared to the control group $(\mathrm{CI}, 80.81 \pm 11.97 \mathrm{U} / \mathrm{ml}$; $\mathrm{ICH}, 80.25 \pm 10.91 \mathrm{U} / \mathrm{ml}$; SAH, 74.43 $\pm 11.39 \mathrm{U} / \mathrm{ml}$ and control, 41.08 $\pm 5.10 \mathrm{U} / \mathrm{ml} ; \mathrm{P}<0.05$ ). Additionally, serum TC, LDL and TG levels significantly increased, while serum HDL significantly decreased in the ACVD groups compared to the control group $(\mathrm{P}<0.05)$. A positive correlation was found between serum IMA levels and serum TC, LDL and TG levels in the ACVD patients, while serum IMA levels were negatively correlated with serum HDL levels $(\mathrm{P}<0.05)$. Thus, serum IMA increased following an ACVD event and was closely correlated with changes in the blood lipid levels. These factors, when combined, may allow the development of molecular markers for an earlier diagnosis of ACVD.
\end{abstract}

\section{Introduction}

Acute cerebrovascular disease (ACVD) is a common disorder of the nervous system that typically affects blood vessels in the brain and mainly includes cerebral infarction (CI), intracerebral hemorrhage (ICH) and subarachnoid hemorrhage

Correspondence to: Professor Kun Han, Department of Neurology, The First Affiliated Hospital of Liaoning Medical College, No. 2 of the Five Duan of Renminjie, Jinzhou, Liaoning 121001, P.R. China E-mail: jinzhouhk@126.com

Key words: acute cerebrovascular disease, blood lipid, correlation, ischemia modified album
(SAH), which can result in severe neurological impairment or death (1). ACVD frequently affects older individuals, resulting in an increase in morbidity and mortality rates each year in China. However, changes to the living environment and diet structures are also key factors that should be considered (2). Notably, early diagnosis and treatment are able to reduce the degree of neurological impairment and mortality resulting from ACVD events (3).

ACVD diagnosis currently relies heavily on neuroimaging (2). However, studies conducted in China and worldwide have focused on the identification of biochemical markers to improve early diagnosis and to distinguish between ischemic and hemorrhagic stroke, similar to the way in which troponin is used to diagnose acute myocardial infarction (2). One biochemical marker, ischemia-modified albumin (IMA), has been widely studied in tissue ischemia in recent years. IMA appears to be an early indicator of myocardial ischemia, which is detectable before the occurrence of myocardial infarction (4-6). Moreover, serum IMA increases in mesenteric thrombosis, pulmonary embolism, stroke and other ischemic diseases (7-11). Thus, in addition to being a predictor of myocardial infarction, IMA may be a useful marker for ACVD.

Increased IMA also appears to be associated with altered lipid profiles. Individuals with hypercholesterolemia demonstrate increased IMA in addition to increased low-density lipoproteins (LDL) (12). Similarly, in cases of obesity or metabolic syndrome, an altered lipid metabolism and an increased IMA are often observed together $(13,14)$. Notably, ACVD patients have been known to exhibit complications of abnormal lipid metabolism (15). Hypercholesterolemia has been identified as an independent risk factor for ACVD (16). While findings of an earlier study demonstrated that IMA could be used to distinguish between certain types of ACVD (11), whether or not IMA levels are associated with lipid profiles in ACVD and whether these markers may be combined to improve diagnosis or treatment of ACVD remain to be determined.

To determine whether IMA and lipid levels are associated with ACVD, serum IMA was measured using the albumin cobalt binding test in 62 subjects with CI, 40 with ICH, 18 with SAH and 100 healthy individuals. In addition, total cholesterol (TC), LDL cholesterol (LDL-C), high-density lipoprotein cholesterol (HDL-C) and triglyceride (TG) levels were determined to in order to explore correlations between serum IMA and lipid levels in ACVD patients. 
Table I. Comparison of characteristics of patients with ACVD vs. healthy individuals.

\begin{tabular}{lcccccr}
\hline & \multicolumn{5}{c}{ ACVD type } \\
\cline { 2 - 4 } Characteristics & CI & ICH & SAH & Control & $\chi^{2}$ or F-value & P-value \\
\cline { 2 - 5 } No. of patients & 62 & 40 & 18 & 100 & & \\
Gender (M/F) & $38 / 24$ & $24 / 16$ & $10 / 8$ & $53 / 47$ & 1.276 & 0.735 \\
Age (years) & $59.18 \pm 8.60$ & $60.93 \pm 8.13$ & $60.44 \pm 9.52$ & $60.83 \pm 8.58$ & 0.55 & 0.648 \\
Body mass index $\left(\mathrm{kg} / \mathrm{m}^{2}\right)$ & $24.74 \pm 3.38$ & $24.68 \pm 2.90$ & $24.45 \pm 1.81$ & $24.12 \pm 3.00$ & 0.663 & 0.576 \\
Blood glucose $(\mathrm{mmol} / \mathrm{l})$ & $5.78 \pm 0.57^{\mathrm{a}}$ & $5.62 \pm 0.43^{\mathrm{a}}$ & $5.65 \pm 0.47^{\mathrm{a}}$ & $5.25 \pm 0.44$ & 17.69 & 0.001 \\
Systolic pressure $(\mathrm{mmHg})$ & $142.10 \pm 11.70^{\mathrm{a}}$ & $147.85 \pm 12.12^{\mathrm{a}}$ & $147.44 \pm 10.04^{\mathrm{a}}$ & $133.52 \pm 7.24$ & 27.155 & 0.001 \\
Diastolic pressure (mmHg) & $85.85 \pm 4.45^{\mathrm{a}}$ & $87.75 \pm 3.95^{\mathrm{a}}$ & $86.67 \pm 3.50^{\mathrm{a}}$ & $82.01 \pm 7.52$ & 11.708 & 0.001 \\
Serum albumin $(\mathrm{g} / \mathrm{l})$ & $39.40 \pm 2.85$ & $39.48 \pm 2.89$ & $39.01 \pm 3.15$ & $38.67 \pm 3.48$ & 0.965 & 0.41 \\
\hline
\end{tabular}

${ }^{a} \mathrm{P}<0.05$ vs. control. AVCD, acute cerebrovascular disease; CI, cerebral infarction; ICH, intracerebral hemorrhage; SAH, subarachnoid hemorrhage.

Table II. Comparison of serum IMA and blood lipid levels among types of ACVD.

\begin{tabular}{|c|c|c|c|c|c|c|}
\hline & \multicolumn{3}{|c|}{ AVCD type } & \multirow[b]{2}{*}{ Control } & \multirow[b]{2}{*}{ F-value } & \multirow[b]{2}{*}{ P-value } \\
\hline & $\mathrm{CI}$ & $\mathrm{ICH}$ & $\mathrm{SAH}$ & & & \\
\hline No. of patients & 62 & 40 & 18 & 100 & & \\
\hline IMA (U/ml) & $80.81 \pm 11.97^{\mathrm{a}}$ & $80.25 \pm 10.91^{\mathrm{a}}$ & $74.43 \pm 11.39^{\mathrm{a}}$ & $41.08 \pm 5.10$ & 324.176 & 0.001 \\
\hline $\mathrm{TC}(\mathrm{mmol} / \mathrm{l})$ & $5.74 \pm 0.19^{\mathrm{a}}$ & $5.42 \pm 0.26^{\mathrm{a}}$ & $5.23 \pm 0.29^{\mathrm{a}}$ & $4.91 \pm 0.06$ & 312.241 & 0.001 \\
\hline TG (mmol/l) & $1.75 \pm 0.21$ & $1.65 \pm 0.20$ & $1.55 \pm 0.17$ & $1.44 \pm 0.15$ & 40.801 & 0.001 \\
\hline LDL-C (mmol/l) & $3.52 \pm 0.36^{\mathrm{a}}$ & $3.17 \pm 0.34^{\mathrm{a}}$ & $3.02 \pm 0.27^{\mathrm{a}}$ & $2.64 \pm 0.23$ & 117.767 & 0.001 \\
\hline HDL-C (mmol/l) & $1.07 \pm 0.16^{\mathrm{a}}$ & $1.16 \pm 0.16^{\mathrm{a}}$ & $1.19 \pm 0.12^{\mathrm{a}}$ & $1.30 \pm 0.17$ & 24.849 & 0.001 \\
\hline
\end{tabular}

${ }^{a} \mathrm{P}<0.05$, vs. control. AVCD, acute cerebrovascular disease; CI, cerebral infarction; ICH, intracerebral hemorrhage; SAH, subarachnoid hemorrhage; IMA, ischemia-modified albumin; TC, total cholesterol; TG, triglycerides; LDL-C, low-density lipoprotein cholesterol; HDL-C, high-density lipoprotein cholesterol.

\section{Subjects and methods}

Subjects. A total of 120 ACVD patients who were hospitalized at the First Affiliated Hospital of Liaoning Medical College (Jinzhou, Liaoning, China) between January 2009 and December 2011 were included in the study. Diagnoses met the criteria established by the 4th National Cerebrovascular Disease Conference in 1995 (17), and were confirmed by CT or MRI. Complete biochemical data were available for all participants. Participants were divided into groups according to the type of ACVD. The CI group included 62 patients, 38 males and 24 females, aged 40-74 years; the ICH group included 40 patients, 24 males and 16 females, aged $44-74$ years; the SAH group included 18 patients, 10 males and 8 females, aged 42-75 years. The exclusion criteria for patients with ACVD were: severe liver or renal failure, major surgery one month prior to disease onset or trauma history, coronary artery thrombosis within six months, chronic inflammatory or infectious diseases, previous history of cerebral apoplexy, pulmonary embolism or thrombotic diseases related to systemic circulation or pregnancy. One hundred healthy individuals who received physical examination at our hospital during the same period were selected as the control group. Participants in the control group had not taken any medicine within two weeks. The study was approved by the Ethics Committee of our hospital, and all enrolled participants or their families provided informed consent.

Biochemistry. Blood samples for the detection of IMA were collected $3 \mathrm{~h}$ after ACVD. Blood samples for the lipid profiles were collected following a 12-h fasting period. Serum was separated and stored at $-70^{\circ} \mathrm{C}$. IMA levels were detected by enzyme-linked immunosorbent assay (ELISA) using a kit from R\&D Systems (Minneapolis, MN, USA). TC, TG, LDL-C and HDL-C levels were measured by enzymatic method (Roche Diagnostics, Indianapolis, IN, USA) using the Beckman LX-20 Automatic Biochemical Analyzer according to the manufacturer's instructions.

Statistical analysis. SPSS 17.0 statistical software was used for statistical analysis. Measurements were presented as the mean \pm standard deviation. The $\chi^{2}$ test was used to compare gender distribution between the groups, and the single-factor ANOVA test was used to compare general characteristics, 
Table III. Correlation between serum IMA and blood lipid levels in ACVD patients.

\begin{tabular}{lcccc}
\hline Correlation & TC & TG & LDL-C & HDL-C \\
\hline r-value & 0.496 & 0.575 & 0.523 & -0.536 \\
P-value & 0.001 & 0.001 & 0.001 & 0.001 \\
\hline
\end{tabular}

IMA, ischemia-modified albumin; ACVD, acute cerebrovascular disease; TC, total cholesterol; TG, triglycerides; LDL-C, low-density lipoprotein cholesterol, HDL-C, high density lipoprotein cholesterol.

serum IMA and lipid levels, as well as to perform a paired comparison (Student Newman-Keuls test). Pearson's correlation method was used to analyze the correlation between serum IMA and lipid levels in the ACVD patients. Analyses were two-sided, with an $\alpha$ level of 0.05 . $\mathrm{P}<0.05$ was considered to indicate a statistically significant difference.

\section{Results}

ACVD demographics. No statistical difference was found between the CI, ICH and SAH and control groups with regard to variables such as gender, age, body mass index and blood albumin (Table I). However, blood glucose, systolic blood pressure and diastolic pressure were significantly higher in all of the ACVD groups compared to the control group $(\mathrm{P}<0.05)$.

Serum IMA and lipid levels. Upon measuring the serum IMA levels as well as the lipid profiles of participants experiencing an ACVD event, we found that serum IMA, TC and LDL-C ('bad' cholesterol) levels were significantly higher in the patients of each ACVD group compared to the control group $(\mathrm{P}<0.05)$. However, HDL-C ('good' cholesterol) levels significantly decreased in individuals with ACVD compared to the controls $(\mathrm{P}<0.05)$ (Table II).

Correlation between serum IMA and lipid levels. To determine whether serum IMA levels in ACVD patients are associated with the observed changes in lipid levels, the Pearson's correlation was used. Serum IMA levels positively correlated with patients' TC, TG and LDL-C levels, but negatively correlated with HDL-C levels $(\mathrm{P}<0.05$; Table III). Serum TC, TG and LDL-C levels significantly increased, while HDL-C levels significantly decreased, with increasing serum IMA levels (Fig. 1).

\section{Discussion}

ACVD accounts for $10 \%$ of mortality among all diseases and is one of the three major causes of mortality in humans (18). Additionally, ACVD events result in high rates of disability due to lasting neurological damage (2). The key to reducing morbidity and mortality associated with these events is early diagnosis and treatment. However, ACVD diagnosis currently relies on clinical symptoms and neuroimaging (2). Molecular markers that are able to improve diagnosis are required to reduce ACVD mortality.
A

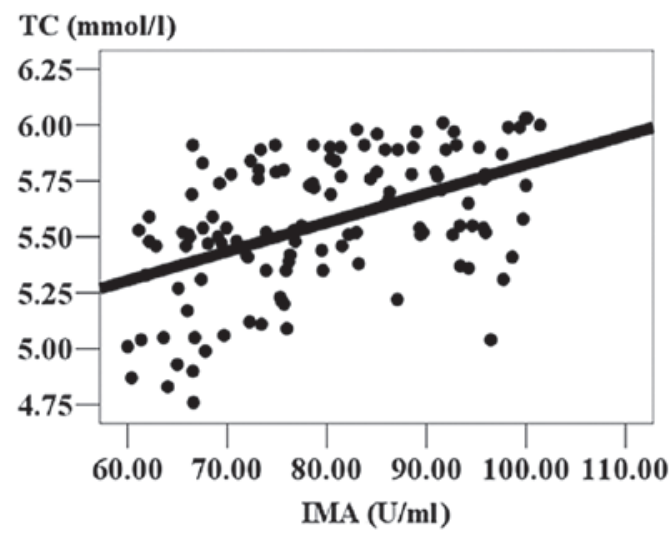

B

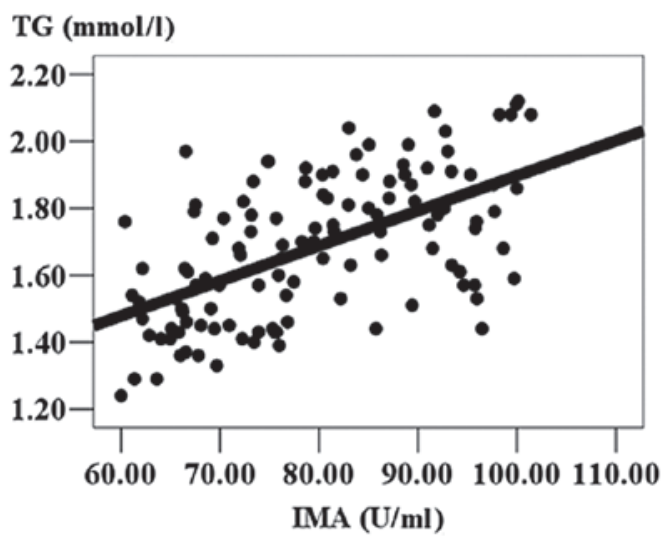

C LDL-C (mmol/l)



D

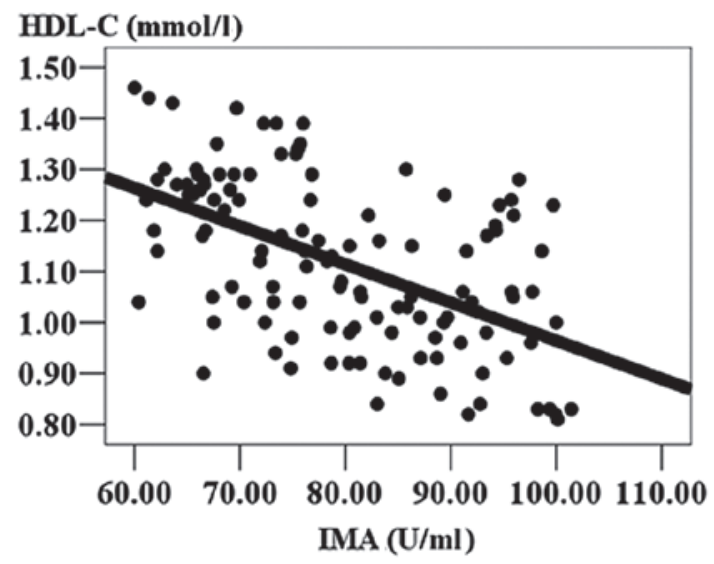

Figure 1. Correlation between serum IMA and blood lipid levels in ACVD patients. IMA, ischemia-modified albumin; ACVD, acute cerebrovascular disease; TC, total cholesterol; TG, triglycerides; LDL-C, low-density lipoprotein cholesterol, HDL-C, high density lipoprotein cholesterol. 
IMA, derived from human serum albumin (HSA), is an important molecular marker in cardiac care due to its association with ischemic events (19). The amino-terminal sequence of HSA, which is unique to humans, is the major binding site of transition metals, including $\mathrm{Co}, \mathrm{Cu}$ and $\mathrm{Ni}$. When HSA is transported through ischemic tissues, free radicals released following ischemia/re-perfusion disrupt the amino-terminal sequence of HSA, reducing its binding capacity with transition metals; the altered HSA is termed IMA (20). IMA increases during acute myocardial ischemia, but may also increase during non-cardiac ischemic events, including infection, cerebrovascular disease, end-stage renal failure and tumor diseases. A recent study demonstrated that serum IMA levels differed according to the type of ACVD event (11). In this study, we found that serum IMA levels in early-stage $(<3 \mathrm{~h}$ following event) ACVD patients were significantly higher compared to the healthy individuals, which is likely to be due to the fact that serum IMA concentrations increased in relation to the ischemic event. Additionally, IMA reflects the degree of ischemia, as demonstrated by results obtained by Abboud et al (21). Thus, IMA may be a useful marker for detecting ischemia correlated with ACVD.

Serum IMA concentrations are affected by a number of factors, particularly serum albumin and serum lipid levels (19). Since other diseases that are closely associated with changes in serum lipids also appear to demonstrate increased IMA (e.g., metabolic syndrome and obesity), there may be a specific link between these features in causing disease $(13,14)$. This observation is emphasized by the fact that patients with pure hypercholesterolemia have increased IMA (12). We observed that IMA levels in ACVD patients were positively correlated with TC, TG and LDL-C concentrations, supporting findings for other diseases. A potential reason for the correlation between IMA and lipid changes in ACVD is that, during ACVD attack, high concentrations of blood lipids increase the local blood viscosity, causing slow blood flow. In turn, platelets aggregate and form blood clots more easily, promoting the progression of ACVD. In addition, high concentrations of serum lipids are able to increase the instability of vulnerable plaques, aggravating artery stenosis or promoting the formation of blood clots, thereby aggravating ischemia and resulting in increased IMA (22). A second possibility is that highly concentrated serum combines with albumin to reduce binding sites of albumin and cobalt ions, causing a decrease in the binding capacity of albumin cobalt, which is manifested as increased levels of serum IMA (23).

In conclusion, an increase in the levels of serum IMA of ACVD patients was observed. This increase renders IMA a useful marker for the early diagnosis of ACVD. Additionally, serum IMA levels in ACVD are closely correlated with lipid levels. The molecular mechanisms behind the correlation between IMA and lipids should be investigated. However, it is possible these mechanisms may be developed into a more sensitive diagnostic tool for ACVD.

\section{References}

1. Martinez-Gonzãlez NA and Sudlow CL: Effects of apolipoprotein E genotype on outcome after ischaemic stroke, intracerebral haemorrhage and subarachnoid haemorrhage. J Neurol Neurosurg Pshychiatry 77: 1329-1335, 2006.
2. Gong X, Fang M, Wang J, et al: Three-dimensional reconstruction of brain surface anatomy based on magnetic resonance imaging diffusion-weighted imaging: a new approach. J Biomed Sci 11: 711-716, 2004.

3. Salvarani C, Brown RD Jr, Calamia KT, et al: Primary central nervous system vasculitis: analysis of 101 patients. Ann Neurol 62: 442-451, 2007.

4. Wu AH: The ischemia modified albumin biomarker for myocardial ischemia. MLO Med Lab Obs 35: 36-38, 2003.

5. Sinha MK, Roy D, Gaze DC, Collinson PO and Kaski JC: Role of 'ischemia modified albumin', a new biochemical marker of myocardial ischemia, in the early diagnosis of acute coronary syndrome. Emerg Med J 21: 29-34, 2004.

6. Sacchetti A: 'Ischemia modified albumin': a new biochemical marker of myocardial ischemia. Emerg Med J 21: 3-4, 2004.

7. Keating L, Benger JR, Beetham R, et al: The PRIM A study: presentation of ischemia modified albumin in the emergency department. Emerg Med J 23: 764-768, 2006.

8. Refaai MA, Wright RW, Parvin CA, et al: Ischemia-modified albumin increases after skeletal muscle ischemia during arthroscopic knee surgery. Clin Chim Acta 366: 264-268, 2006.

9. Turedi S, Gunduz A, Mentese, et al: The value of ischemiamodified albumin in the diagnosis of pulmonary embolism. AM J Emerg Med 25: 770-773, 2007.

10. Gunduz A, Turedi S, Mentese A, et al: Ischemia-modified albumin in the diagnosis of acute mesenteric ischemia: a preliminary study. AM J Emerg Med 26: 202-205, 2009.

11. Gunduz A, Turedi S, Mentese A, et al: Ischemia-modified albumin levels in cerebrovascular accidents. Am J Emerg Med 26: 874-878, 2008.

12. Duarte MM, Rocha JB, Moresco RN, et al: Association between ischemia-modified albumin, lipids and inflammation biomarkers in patients with hypercholesterolemia. Clin Biochem 42: 666-671, 2009.

13. Valle Gottlieb MG, da Cruz IB, Duarte MM, et al: Associations among metabolic syndrome, ischemia, inflammatory, oxidatives, and lipids biomarkers. J Clin Endocrinol Metab 95: 586-591, 2010.

14. Piva SJ, Duarte MM, Da Cruz IB, et al: Ischemia-modified albumin as an oxidative stress biomarker in obesity. Clin Biochem 44: 345-347, 2011.

15. Velcheva I, Antonova N, Dimitrova V, et al: Plasma lipids and blood viscosity in patients with cerebrovascular disease. Clin Hemorheol Microcirc 35: 155-157, 2006.

16. Kimura Y and Uchiyama S: Hyperlipidemia and cerebrovascular disease. Nihon Rinsho 23: 685-689, 2001.

17. Moore, WS, Barnett HJ, Beebe HG, et al: Guidelines for carotid endarterectomy. A multidisciplinary consensus statement from the Ad Hoc Committee, American Heart Association. Circulation 91: 566-579, 1995.

18. Pappachan J and Kirkham FJ: Cerebrovascular disease and stroke. Arch Dis Child 93: 890-898, 2008.

19. Roy D, Quiles J, Sharma R, et al: Ischemia-modified albumin concentrations in patients with peripheral vascular disease and exercise-induced skeletal muscle ischemia. Clin Chem 50: 1656-1680, 2004.

20. Lippi G, Montagnana M and Guidi GC: Albumin cobalt binding and ischemia modified albumin generation: an endogenous response to ischemia. Int J Cardiol 108: 410-411, 2006.

21. Abboud H, Labreuche J, Meseguer E, et al: Ischemia-modified albumin in acute stroke. Cerebrovasc Dis 23: 216-220, 2007.

22. Wei LX, Tang Q H, Sun L, et al: Relationship between oxidized lipoprotein, angiogenesis and human coronary atherosclerotic plaque stabilization. Zhonghua Bing Li Xue Za Zhi 35: 138-141, 2006.

23. Bhagavan NV, Ha JS, Park JH, et al: Utility of serum fatty acid concentrations as a marker for acute myocardial infarction and their potential role in the formation of ischemia-modified albumin: a pilot study. Clin Chem 55: 1588-1590, 2009. 